Background Dementia is commonly associated with co-morbidities and non-specific presentations. Individualised dementia care should ideally be provided within the context of these co-morbidities and presentations. To provide systematic insights, we described the dynamic changes of 20 commonest healthcare visits prior to the diagnosis dementia in Taiwan.

Methods We adopted a nested case-control approach. From the Taiwanese healthcare records, which covers $>99 \%$ of Taiwanese population, we identified 491,000 case individuals first diagnosed to have dementia after age 50 years during 2002-2015, and control individuals matched at 1:1 ratio by age, sex, year of birth, and region at the time of first diagnosis. By 3-digit codes according to the International Classification of Diseases, 9th Edition (ICD-9), we identified 20 commonest causes of healthcare visits in case individuals between 2000 and 2015. Using stratified Poisson regression, we estimated cause-specific rates of healthcare visits between cases and controls within a compatible person-year, and observed the change of relative rates over the 8-year span leading to the diagnosis of dementia. With the large sample size, confidence of intervals of these relative rates for common events were almost negligible.

Results There was gradual increase in average numbers of allcause healthcare visits prior to the diagnosis of dementia. However, we observed various decline of 15 of 20 cause-specific care prior to the diagnosis of dementia compared to controls, including diabetes mellitus (ICD-9 250), disorders of lipid metabolism (ICD-9 272), cataract (ICD-9 366), disorders of conjunctiva (ICD-9 372), essential hypertension (ICD-9 401 ), other form of chronic ischaemic heart disease (ICD-9 414), acute nasopharyngitis (ICD-9 460), acute upper respiratory infections of multiple or unspecific sites (ICD-9 465), acute bronchitis and bronchiolitis (ICD-9 466), diseases of hard tissues of teeth (ICD-9 521), and gingival and periodontal diseases (ICD-9 522), gastritis and duodenitis (ICD-9 535), osteo-arthrosis and allied disorders (ICD-9 715), other and unspecified disorders of back (ICD-9 724), and other disorders of soft tissues (ICD-9 729). Four causes of healthcare visits that increased prior to the diagnosis of dementia included neurotic disorders (ICD-9 300), disorders of function of stomach (ICD-9 536) functional digestive disorders, not elsewhere specified (ICD-9 564), and possibly contact dermatitis and other eczema (ICD-9 692). There was little change in hypertensive heart disease (ICD-9 401 prior to the diagnosis of dementia)

Conclusion Paradoxical to the increase in all-cause healthcare visits prior to the diagnosis of dementia, there was decline of commonest care prior to the diagnosis of dementia. The few increasing exceptions could be common presentations prior to the diagnosis of dementia. These time-dependent findings provide systematic insights into dementia care and diagnosis.

\section{P30 TREND IN DEMENTIA INCIDENCE IN CHINA 2002-2014: POPULATION-BASED LONGITUDINAL STUDY}

${ }^{1}$ Yixin Li* ${ }^{*}{ }^{2}$ Marzieh Araghi, ${ }^{3}$ Jing Liao, ${ }^{4}$ Archana Singh-Manoux, ${ }^{1}$ Eric Brunner. ${ }^{1}$ Institute of Epidemiology and Health Care, University College London, London, UK; ${ }^{2}$ School of Public Health, Epidemiology and Biostatistics, Imperial College London, London, UK; ${ }^{3}$ Department of Medical Statistics and Epidemiology | Sun Yat-sen University, Sun Yat-sen University, Guangzhou, China; ${ }^{4}$ Inserm U1153, Epidemiology of Ageing and Neurodegenerati, University de Paris, Paris, France
Background Globally, some 50 million people had Alzheimer's disease and related dementias in 2018. China accounts for approximately a quarter of cases. It is estimated that the cost of dementia in China was approximately USD 50 billion in 2010. China's rapid ageing process is occurring at an earlier stage of economic development than other countries, posing great demands on society, particularly health and care services. Accurate estimation of the recent time trend in the incidence of dementia in China is critical for tackling future challenges and formulating public health policies. There is evidence from several high-income countries of a recent downward trend in dementia incidence rates. It is currently unclear, in view of its less-developed status, whether the time trend is also downward in China. The aim of this study is to estimate the recent time trend in dementia incidence in China and to explore how much the effect of changes in risk factors on this trend.

Methods The Chinese Longitudinal Healthy Longevity Study (CLHLS) provides five waves of data (2002-2014). CLHLS wave 3 to wave 8 provided information about health status and quality of life of 47,584 people aged over 60 years in 23 provinces, municipalities and autonomous regions of China. In order to maintain representativeness, refreshment participants were recruited to study periodically. Dementia was ascertained at each wave using standardized cognitive and functional impairment criteria. Competing risks of mortality and non-random dropout were accounted for in analysing the temporal trend in dementia incidence. Joint model was fitted to estimate the temporal trends in dementia incidence in China. $\mathrm{R}$ package JM was employed to fitted the joint model.

Results Age-sex adjusted dementia incidence (2002-2014) increased at an annual rate of $2.5 \%$ (95\% CI $2.4 \%-2.6 \%$ ). Changes in potential risk factors accounted for about $20 \%$ of the time effect in dementia incidence.

Conclusion Time trends in dementia incidence in China was upward recently. To our knowledge, this is the first study to estimate the trend in dementia incidence in a middle-income country. This longitudinal study used joint modelling with time-to-event data to explore the time trends in dementia incidence for 12 years in China. The first limitation of this study was not possess data from eight provinces in remote areas in China. The second potential limitation is caused by the recall bias and measurements bias over time. The third limitation is that we assumed a constant rate of change of the dementia incidence rate which based on the rate of change was uniform across the Chinese population, but the population structure changed between 2002 to 2014 .

\section{P31 ABSTRACT WITHDRAWN}

\section{P32 AORTIC STIFFNESS AS A RISK FACTOR FOR DECLINE IN PHYSICAL FUNCTIONING IN THE WHITEHALL II STUDY}

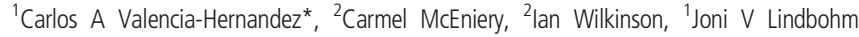
${ }^{1}$ Sara Ahmadi-Abhari, ${ }^{1}$ Martin J Shipley, 'Eric J Brunner. 'Epidemiology and Public Health, University College London, London, UK; ${ }^{2}$ Division of Experimental Medicine and Immunotherapeutics, University of Cambridge, Cambridge, UK 